\title{
Novel Waveforms, Models, Algorithms for Cable Health Monitoring
}

\author{
Xuan Wang ${ }^{1}$ \\ ${ }^{1}$ University of South Carolina, Columbia, South Carolina, 29208, United States \\ xuanw@email.sc.edu
}

\begin{abstract}
Cables in power generation and delivery are under high thermal stress cycles. Such high temperature can lead to cable insulation degradation, which will reduce the projected lifetime. Existing methods mainly focus on cable fault detection or insulation degradation mechanism. There is no existing tools for diagnosing the insulation degradation level and predicting the remaining useful life of the cable. The goal of my Ph.D. research is to develop reflectometry and data based approaches to monitor the health status of cables. The research will be conducted in three steps: (1) development of reflectometry based method to monitor the cable insulation degradation; (2) feature extraction and cable insulation degradation dynamic modeling based on the accelerated aging test data; (3) development of risk-senstive particle filtering based fault diagnosis and prognosis algorithms for cable degradation; and (4)Verification and validation the proposed solution with new experiment data and comparison with existing approaches.
\end{abstract}

\section{Problem Statement}

Long-term exposure to radiation, thermal and other adverse environmental conditions is the leading causes of damage and embrittlement of cable insulation, which could lead to economic and safety concerns for the reliable operation of power plants and power delivery (Shimada, Sugimoto, Kudoh, Tamura, \& Seguchi, 2014). Insulation damage can occur due to overheating caused by the high current flow through the conductor. If the grounded conductor is close to the insulated cable, at high voltage a significant amount of current may flow through the insulation causing heating and erosion of insulation. Sensitive nondestructive evaluation (NDE) techniques are important in detecting cable insulation degradation and predicting the remaining useful life (RUL) in cables so that the maintenance and replacement of cables can be optimized, which saves cost and assure cable performance.

The quantitative analysis of degradation level of insulation is a challenging and open problem. Due to this limitation, the predication of RUL of cable remaining unsolved. There are some existing method to detect degradation of cable insulation. Although these methods show some successes, each method however has its own advantages and limitations. Elongation at break (EAB) [2] and compressive modulus are directly related with the physical property of the cable insulation. They are either destructive or need access to the cable

\footnotetext{
${ }^{1}$ Xuan Wang. This is an open-access article distributed under the terms of the Creative Commons Attribution 3.0 United States License, which permits unrestricted use, distribution, and reproduction in any medium, pro-vided the original author and source are credited.
}

surface which is not desirable or even impossible for some applications. Oxidation induction time can be only performed in the laboratory settings. Time domain reflectometry (TDR) is sensitive to incipient fault but can not provide information about the future performance. Partial discharge (PD) needs to apply a relatively high voltage to the cable under test, which may aggravate the insulation degradation.

To address these limitations, my research introduces several innovative new concepts and integrates them from an interdisciplinary point of view. The key innovative concepts include new waveforms and diagnostic methods for insulation defect testing, probabilistic algorithm development and statistical decision thresholds for cable fault determination.

Joint Time-Frequency Domain Reflectometry (JTFDR) waveforms will be developed to detect insulation damage in cables that are subjected to accelerated aging tests(Wang, Stone, Coats, Shin, \& Dougal, 2011). The primary focus is to develop JTFDR waveforms with certain center frequency and frequency bandwidth that are sensitive to insulation damage. Continuous wavelet transform (CWT) will be used to get a time-frequency overview of signal, thus enabling feature extraction for cable insulation degradation detection. Cable insulation fault model can be developed based on the test data. To accurately estimate and predict the remaining service life of a cable, a particle filtering-based approach is proposed which offers a compromise between data-driven and model-based techniques (Zhang et al., 2011). For verification and validation, high resolution $\mathrm{X}$-ray images of cables that have undergone accelerated aging will be taken and compare with the estimaton from the proposed algorithms.

\section{ExpeCted Contributions}

The contributions of this Ph.D. research include:

(1) Develop a reflectometry based method to monitor the cable insulation degradation process in the accelerated aging test; Integrate it with some signal processing methods to extract features for fault diagnosis.

(2) Conduct accelerated aging test on a large group of cables; build a dynamic model for cable degradation based on the test data.

(3) Develop a risk-sensitive particle filtering method for cable insulation fault diagnosis and prognosis; quantify the uncertainties and analyzing the sensitivity of model parameters.

(4) Verify and validate the proposed solution with new experiment data and compare with existing approaches,such as $\mathrm{EAB}, \mathrm{PD}$, etc. 


\section{Research Plan}

\subsection{Work performed}

\subsubsection{JTFDR Sensing and Monitoring}

In JTFDR a chirp signal with a Gaussian envelope is used. Fig. 1 shows an incident and a reflected JTFDR waveforms along with the cross-correlation. The cross-correlation shows the location of the damage. Similar to TDR, JTFDR waveforms can also be injected directly into the cable or through surface wave or other form of coupling. Typical waveform parameters include the center frequency and frequency bandwidth, which determine the signal attenuation as well as measurement resolution. When surface wave launchers or sensors are used to launcher the incident waves to a certain type of insulation, the waveforms must be designed to allow the frequency band of interest to propagate without significant attenuation over the cable under test. Very recently cases of cable insulation damage (XLPE unshielded) under an accelerated aging condition were investigated(Alam, 2014). The test conditions for accelerated aging are governed by the Arrhenius model for accelerated aging,

$$
\frac{t_{s}}{t_{a}}=e^{\frac{E_{a}}{B}\left(\frac{1}{T_{s}}-\frac{1}{T_{a}}\right)}
$$

where $T_{s}$ is the service temperature ${ }^{\circ} K, T_{a}$ is the accelerated aging temperature ${ }^{\circ} K, t_{s}$ is the aging time in hours at service temperature, $t_{a}$ is the aging time in hours at acceleration temperature, $E_{a}$ is the activation energy of the insulation material (1.33eV for XLPE), $B=1.3810^{-23} \mathrm{~J} /{ }^{\circ} \mathrm{K}=$ $8.61710^{-5} \mathrm{eV} /{ }^{\circ} \mathrm{K}$ is Boltzmanns constant.

Thus, the accelerated aging temperature is

$$
T_{a}=\left[\frac{1}{T_{s}}-\frac{B}{E_{a}} \ln \left(t_{s} / t_{a}\right)\right]^{-1}
$$

The JTFDR waveforms will be generated with center fre-

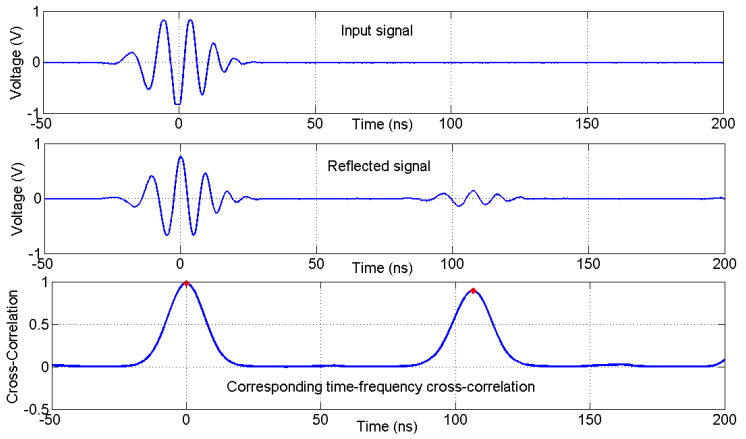

Figure 1. JTFDR signal, $100 \mathrm{MHz}$ center frequency.Input signal,output signal and corresponding cross-correlation

quency ranging from 50-300 $\mathrm{MHz}$. Other paramters, such as frequency span and signal amplitude will be optimized for different cables. Cable insulation damage will be created for a set of different cables with different types, lengths, and fault locations using the accelerated aging method, JTFDR will then be taken for all cable specimens for analysis.

\subsubsection{Accelerated Aging Test of Cable}

A cable aging test platform has been set up as shown in Fig. 3 . In this scheme, the incident waveform is generated in the computer, which is sent to the signal generator to generate the desired waveform. The signal generator sends the proposed incident signals to the oscilloscope and cable through a signal circulator. The incident signal will be reflected back to the oscilloscope when it reaches impedance mismatch in the cable. All signal data, including incident signal and reflected signal, will be stored and analyzed.

In the test,a bunch of JTFDR signal that have the same time duration of $100 \mathrm{~ns}$ and same bandwidth of $100 \mathrm{MHz}$ are designed. The differences are that these signals have different center frequencies of $80 \mathrm{MHz}, 90 \mathrm{MHz}, 100 \mathrm{MHz}, 110 \mathrm{MHz}$, $120 \mathrm{MHz}, 200 \mathrm{MHz}$ and $400 \mathrm{MHz}$ respectively. In the aging

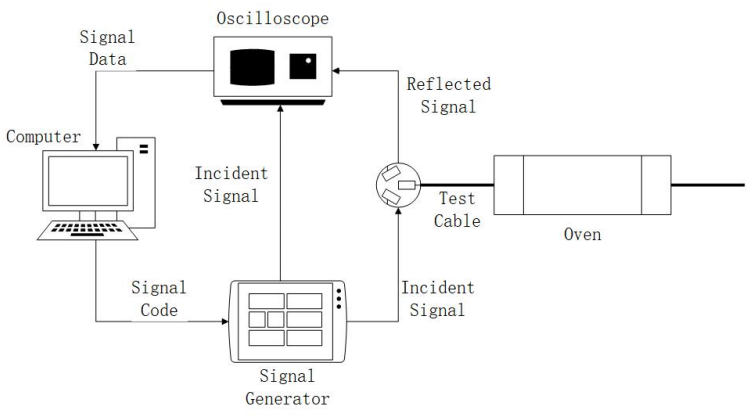

Figure 2. Cable aging test platform.

process, about 0.8 meter of a 20 -meter-long cable is put into the oven. This part of the cable is heated 8 hours a day at $120{ }^{\circ} \mathrm{C}$. After the heating process, the cable is put at room temperature around $20^{\circ} \mathrm{C}$ for cooling. The signal data are collected each day before the heating process at around 20 ${ }^{\circ} \mathrm{C}$.

\subsubsection{Continuous Wavelet Transform for Feature Ex- traction}

Continuous Wavelet Transform (CWT) is a very powerful tool to obtain time frequency representation, which links a one-dimensional time signal to a two-dimensional function of time and frequency(Feizifar, Haghifam, \& Soleymani, 2012). Because JTFDR signals have both time and frequency information, CWT is used to provide a comprehensive analysis of the JTFDR signal. Figure 3 shows the CWT of the JTFDR signal. The oval shadow in the black rectangle is the reflection from the heated area. As the experiment goes on, the area of the shadow will grow up and the color corresponding to magnitude will become larger, which indicates that the reflection becomes stronger.

The magnitude scalogram shown in Fig. 3 is a matrix with size of 71 by 1004 . Each row corresponds to a certain frequency and each column corresponds to a certain time instant. By creating a window that covers the heated area reflection, a smaller matrix that represents the reflection magnitude value can be extracted. The insulation degradation level can be quantified by the extracted matrix.

Based on the matrix, three features are defined to represent the insulation degradation level of the aged area. The three 


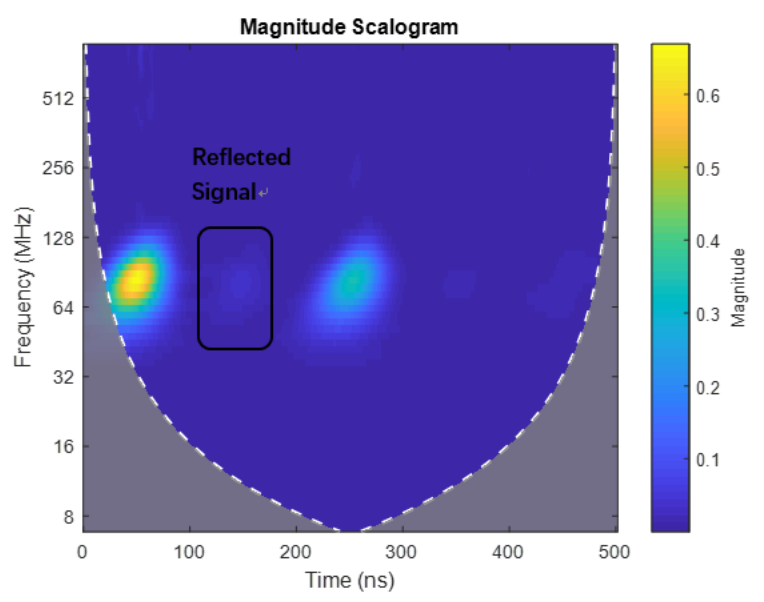

Figure 3. CWT of the JTFDR signal.

features are Sum of Absolute Values (SAV) and Infinite Norm of the Matrix (INM).

$$
\begin{gathered}
S A V=\sum_{i, j=1}^{n}\left|X_{i j}\right| \\
I N M=\max _{1 \leq i \leq m} \sum_{j=1}^{n}\left|a_{i j}\right|
\end{gathered}
$$

As the insulation degrades, the feature value will increase due to stronger reflection. Figure 4.shows the two features change along with experimental days. It shows that these feature can indicate the degradation of cable insulation and can be used for fault diagnosis and prognosis.
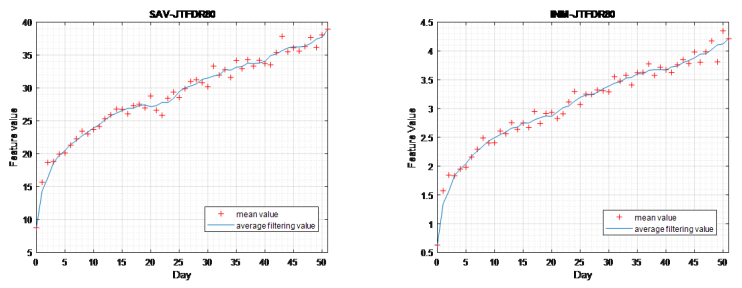

Figure 4. Feature growth with time; a). SAV b). INM

\subsection{Remaining work}

\subsubsection{Cable insulation degradation model}

The feature extracted from the JTFDR signal can well represent the insulation degradation trend. Multiple cables will be tested under the same condition. Based on the experiment data, a model will be established.

\subsubsection{Risk-Sensitive particle filter for cable insulation di- agnosis and prognosis}

Based on the model, We propose a risk-sensitive particle filter (RSPF) algorithm for cable insulation diagnosis and prognosis. The RSPF will be realized by integrating a risk model in the importance distribution to generate more particles in the high-risk regions of the state-space. This is very useful for cable fault diagnosis, which has high risk for power delivery systems.

\subsubsection{Uncertainty management}

The uncertainties of prognosis are represented in the form of a probability density function (PDF) and measured by confidence intervals. The prediction confidence is tied to how the area of this PDF is divided. For sensitivity analysis, extensive experiments will be conducted to establish the relationship between these uncertainty sources and cable degradation.

\subsubsection{Algorithm verification and validation}

The verification and validation will be conducted in two aspects. The first one is to develop the proposed algorithm and compare them with representative existing approaches, such as EAB and PD. The second one is to implement the proposed algorithm on real experiments to test the accuracy of diagnosis and prognosis.

\section{Conclusion}

The goal of this Ph.D research is to develop reflectometry and data based cable health monitoring method. The proposed method aims to overcome the bottleneck of traditional methods in dealing with insulation degradation level quantification. The advantage of these approaches also include better accuracy and precision in cable fault diagnosis and prognosis. The proposed researches will be completed in the next 3 years as the main theme of the author's PhD dissertation.

\section{REFERENCES}

Alam, M. N. (2014). Applications of electromagnetic principles in the design and development of proximity wireless sensors. Dissertations Theses - Gradworks.

Feizifar, B., Haghifam, M. R., \& Soleymani, S. (2012). Application of continuous wavelet transform for fault location in combined overhead line and cable distribution networks. In International conference on electrical electronics engineering.

Shimada, A., Sugimoto, M., Kudoh, H., Tamura, K., \& Seguchi, T. (2014). Degradation mechanisms of silicone rubber (sir) by accelerated ageing for cables of nuclear power plant. IEEE Transactions on Dielectrics Electrical Insulation, 21(1), 16-23.

Wang, J., Stone, P. E. C., Coats, D., Shin, Y. J., \& Dougal, R. A. (2011). Health monitoring of power cable via joint time-frequency domain reflectometry. IEEE Transactions on Instrumentation Measurement, 60(3), 1047-1053.

Zhang, B., Sconyers, C., Byington, C., Patrick, R., Orchard, M. E., \& Vachtsevanos, G. (2011). A probabilistic fault detection approach: Application to bearing fault detection. IEEE Transactions on Industrial Electronics, 58(5), 2011-2018. 